\title{
Computer Simulation of Electric Fields at the Junction Terminal Crest - Pectinate Muscle
}

\author{
G Plank ${ }^{1}$, M Monserrat ${ }^{1}$, J Saiz $^{1}$, E Hofer $^{2}$ \\ ${ }^{1}$ Dpto. Ingeniería Electrónica, Universidad Politecnica de Valencia, Spain \\ ${ }^{2}$ Medizinische Physik und Biophysik, Karl Franzens University Graz, Austria
}

\begin{abstract}
In a previous work we studied the behavior of the electric field $\mathbf{E}$ parallel to a cardiac tisue surface during continuous conduction [1]. We found that 1) the peak field $\hat{\mathbf{E}}$ points opposite the direction of propagation, 2) the instant $T_{E}$ of $\hat{\mathbf{E}}$ represents an accurate time marker of local activation, and 3) vector-loops of $\mathbf{E}$ can be measured using ultradense four-element electrode arrays $(<100 \mu \mathrm{m})$ and high sampling frequencies $(>100 \mathrm{kHz}$ ). Experimentally during recordings in the region Terminal Crest - Pectinate Muscles of rabbit atria we observed loop morphologies, however, which deviated in some instances significantly from the expected behavior. We supposed that, apart from discrete effects, branching and the curvature of the tissue might play a role. Hence we examined by means of a computer model if properties of $\mathbf{E}$, observed in the uniform case, remain unchanged in case of more complex activation patterns at a bifurcation site with curved fibers.
\end{abstract}

\section{Introduction}

Measurements of potentials in the volume conductor surrounding cardiac tissue are widely used to determine the activation pattern within the tissue. Predominantly uni-, bi- and multi-polar potential recordings are reported in the literature, measurements of the electric field $\mathbf{E}$ are rarely found $[2,3]$. Conduction velocity, direction of propagation and contours of wavefront are essential parameters for the assessment of the excitation spread under normal and abnormal conditions. Uni- and bipolar recordings can provide information on the activation time, but conduction velocity or direction of propagation can not be determined. For that purpose multi-electrode mapping systems are used to construct isochrone maps from a set of unipolar recordings giving an overview of the activation sequence. The same information, just for a single site, might be obtained with less technical expense using measurements of $\mathbf{E}$ with a four-element electrode array which yields a very local view of the activation sequence. It is known that due to the high localility of field measurements the suppression of remote activities is quite good (proportional to $1 / r^{2}$ ) and consequently it is expected that parameters obtained from $\mathbf{E}$ reflect accurately the local excitation process.

In this study we simulated excitation spread in an atrial tissue sheet at a junction Terminal Crest (TC) - Pectinate Muscle (PM) and compared the intracellularly obtained parameters direction of propagation, activation time and conduction velocity with those obtained extracellularly from simulated measurements of the electric field $\mathbf{E}$.

\section{Methods}

\subsection{The computer model}

A monodomain computer model was developed consisting of a two-dimensional sheet of excitable tissue surrounded by an unbounded volume conductor. The governing equation is given by

$$
I_{m}=\frac{1}{\beta}\left[\frac{1}{\rho_{i x}} \frac{\partial^{2} V_{m}}{\partial x^{2}}+\frac{1}{\rho_{i y}} \frac{\partial^{2} V_{m}}{\partial y^{2}}\right]=I_{i o n}+c_{m} \frac{\partial V_{m}}{\partial t},
$$

where $I_{m}$ is the transmembrane current density $(\mu A$. $\mathrm{cm}^{-2}$ ), $\beta$ the surface-to-volume ratio (set to $2 / a$ with $a=12 \mu \mathrm{m}), \rho_{i x}$ and $\rho_{i y}$ the intracellular resistivities $(0.25$ and $2.25 \mathrm{k} \Omega \cdot \mathrm{cm}), V_{m}$ the transmembrane potential $(m V), I_{i o n}$ the total ionic current density $\left(\mu \mathrm{A} \cdot \mathrm{cm}^{-2}\right)$, and $c_{m}$ the specific membrane capacitance $\left(1 \mu \mathrm{F} \cdot \mathrm{cm}^{-2}\right)$. Discretization steps in space and time were $25 \mu \mathrm{m}$ and $2.5 \mu s$. The dynamic membrane behavior was described with the Nygren model[4].

We simulated excitation spread in a rectangular tissue sheet $\left(6 \times 1.5 \mathrm{~mm}^{2}\right)$. The PM was insulated electrically from the CT by introducing no flux boundary conditions. Longitudinal elliptic propagation was elicited by pacing the lower left corner of the sheet (see fig. 1).

\subsection{Geometric mapping}

The rectangular region representing the PM was mapped from the mesh $\mathbf{x}=\mathbf{x}(x, y)$ to a curved mesh $\tilde{\mathbf{x}}=\tilde{\mathbf{x}}(x, y)$. We bent each fiber $j$ around a circle with radius $r=r_{0}+$ 
$j \cdot d y$. Then each discrete fiber element is seen under the angle $\varphi=(i \cdot d x) / r$. The new mesh $\tilde{\mathbf{x}}$ is given by:

$$
\begin{aligned}
& \tilde{x}(i, j)=\xi_{0}+r \cdot \sin (\varphi) \\
& \tilde{y}(i, j)=\zeta_{0}-r \cdot(1-\cos (\varphi))
\end{aligned}
$$

This mapping preserves both, the fiber length as well as the inter-fiber distance, and thus does not change neither source density nor conduction velocity.

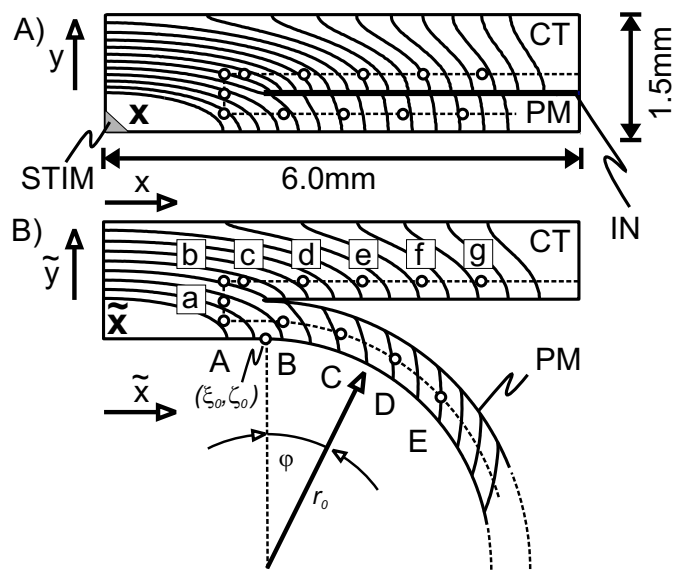

Figure 1. A) Tissue sheet with insulation (IN) between CT and PM and stimulus site (STIM). B) For the computation of $\mathbf{E}$ we mapped the mesh $\mathbf{x}$ onto $\tilde{\mathbf{x}}$. A-E and a-g: observation sites.

\subsection{The electric field $\mathrm{E}$}

The extracellular potential $\Phi_{e}$ and the electric field $\mathbf{E}$ at the observation sites $\mathbf{p}$, designated with $a-f$ in the CT and $A-E$ in the PM, were calculated with

$$
\Phi_{e}(\mathbf{p}, t)=\frac{1}{4 \pi \sigma_{e}} \int \frac{I_{m}(\tilde{\mathbf{x}}, t)}{|\mathbf{r}(\tilde{\mathbf{x}}-\mathbf{p})|} d \Gamma,
$$

and

$$
\mathbf{E}(\mathbf{p}, t)=-\nabla \Phi_{e}(\mathbf{p}, t)=\frac{1}{4 \pi \sigma_{e}} \int \frac{I_{m}(\tilde{\mathbf{x}}, t) \cdot \mathbf{r}}{|\mathbf{r}(\tilde{\mathbf{x}}-\mathbf{p})|^{3}} d \Gamma
$$

where $\mathbf{p}$ is the point of observation, $\tilde{\mathbf{x}}$ the source point, $|\mathbf{r}|$ the distance between $\mathbf{p}$ and $\tilde{\mathbf{x}}, \sigma_{e}$ the conductivity of the homogeneous volume conductor $\left(\mathrm{mS} \cdot \mathrm{cm}^{-1}\right)$, and $\Gamma$ is the surface area of the tissue.

Measurements of $\mathbf{E}$ with four-element electrode arrays were simulated using the following discrete approximation

$$
\tilde{\mathbf{E}}(\mathbf{p}, t)=-\frac{1}{D D} \cdot\left[\begin{array}{c}
\Phi_{e_{2}}(t)-\Phi_{e_{1}}(t) \\
\Phi_{e_{4}}(t)-\Phi_{e_{3}}(t)
\end{array}\right]
$$

where $\Phi_{e_{1-4}}$ represent the potentials at the recording electrodes of a square four-element electrode array. The electrodes 1-2 were oriented parallel to the axis $x$, the electrodes 3-4 parallel to the axis $y$, both electrode pairs with a dipole diameter DD of $60 \mu \mathrm{m}$.

\subsection{Direction of propagation}

The direction of propagation was determined intracellularly by computing first the slope of the tangent to the local isochrone and then taking the negative inverse of the slope as direction of propagation $\vartheta$. We compared $\vartheta$ with the direction obtained from $\vartheta_{E}=\arg (\hat{\mathbf{E}})$ by computing the differences $\Delta \vartheta=\vartheta-\vartheta_{E}$.

\subsection{Activation times}

We compared the extracellular time marker $T_{E}$, i.e. the instant of the peak field $\hat{\mathbf{E}}$, with the intracellularly obtained time marker $T_{I_{m}}$, the instant of maximum negative rate of rise of $I_{m}$, by computing the difference $\Delta T=T_{I_{m}}-T_{E}$ (see fig. 2).
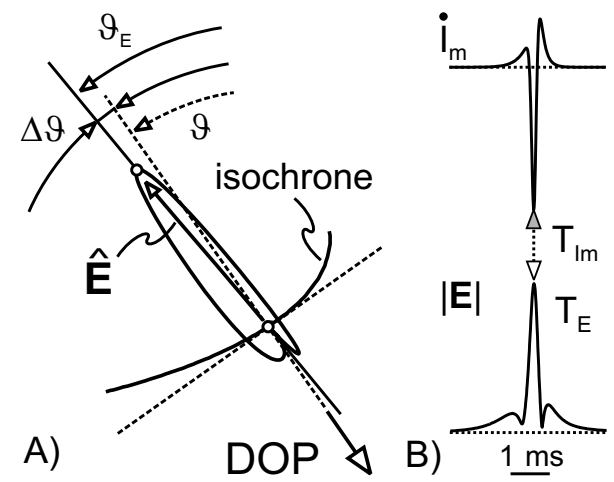

Figure 2. A) Direction of propagation $\vartheta$ (orthogonal to isochrone) was compared with the direction $\vartheta_{E}$ (direction of maximum field $\hat{\mathbf{E}})$. B) Determination of intra- $\left(T_{I_{m}}\right)$ and extracellular time marker $\left(T_{E}\right)$.

\subsection{Conduction velocity}

Conduction velocities were determined in two different ways. Intracellularly we computed the velocity $v_{i}$ by determining the distance between two isochrones with an isochrone interval of $\pm 50 \mu \mathrm{s}$ around the activation time (determined from the instant of the maximum negative derivative of $I_{m}$ ) at the observation site and then dividing this distance by the isochrone interval. Extracellularly we determined the activation times $T_{1-4}$ at the four electrode sites by taking the instant of the maximum negative derivative of $\Phi_{e}$ [5]. With these four samples of the 
activation function $T(x, y)$ we computed the gradient of $T$ at the center of the electrode with a finite difference approach [6]:

$$
\nabla T(x, y)=\left[\begin{array}{c}
T_{x} \\
T_{y}
\end{array}\right]=\frac{1}{D D}\left[\begin{array}{l}
T_{2}-T_{1} \\
T_{4}-T_{3}
\end{array}\right]
$$

Then local conduction velocity $\mathbf{v}$ can be written as

$$
\mathbf{v}=\left[\begin{array}{l}
v_{x} \\
v_{y}
\end{array}\right]=\frac{1}{T_{x}^{2}+T_{y}^{2}}\left[\begin{array}{c}
T_{x} \\
T_{y}
\end{array}\right] ; \quad v=\sqrt{v_{x}^{2}+v_{y}^{2}}
$$

We compared $v$ with $v_{i}$ by computing the differences $\Delta v=v_{i}-v$.

\section{Results and conclusion}

We found at all observation sites good agreement between the parameters obtained from the intracellular isochrones and those obtained from simulated extracellular measurements of the electric field $\mathbf{E}$. As can be seen in fig. 3, vector-loops point opposite the direction of propagation, i.e. they are perpendicular to the local isochrone (maximum deviation $\Delta \vartheta=9.1^{\circ}$ ).

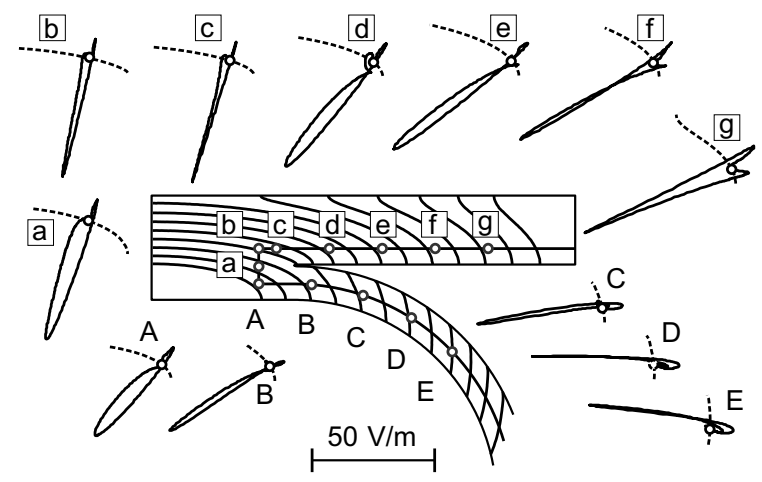

Figure 3. The maximum field $\hat{\mathbf{E}}$ indicates direction of propagation, i.e. vector-loops are perpendicular to local isochrone (dashed line).

Furthermore the time markers $T_{I_{m}}$ and $T_{E}$ coincided closely and the conduction velocities obtained from the four-element electrode array came very close (within the limits of uncertainty due to the sampling interval) to those which were determined from the intracellular isochrone pattern. Differences between extra- and intracellularly obtained parameters are summarized in the following table:

\begin{tabular}{|r||r|c|c|}
\hline & $\Delta v[\%]$ & $\Delta \vartheta\left[^{\circ}\right]$ & $\Delta T[\mu s]$ \\
\hline \hline $\mathrm{a}$ & 1.2 & 1.7 & $\mathbf{5 0}$ \\
\hline $\mathrm{b}$ & -6.7 & -0.9 & 35 \\
\hline $\mathrm{c}$ & -1.5 & -0.2 & 45 \\
\hline $\mathrm{d}$ & -8.5 & 2.4 & 15 \\
\hline $\mathrm{e}$ & -12.0 & 5.1 & 0 \\
\hline $\mathrm{f}$ & $\mathbf{- 1 2 . 1}$ & 2.3 & -10 \\
\hline $\mathrm{g}$ & 6.0 & 1.5 & -5 \\
\hline $\mathrm{A}$ & -11.8 & 6.5 & 35 \\
\hline $\mathrm{B}$ & -6.6 & 5.3 & -10 \\
\hline $\mathrm{C}$ & -11.5 & 2.6 & 5 \\
\hline $\mathrm{D}$ & -2.8 & 6.5 & -5 \\
\hline E & -5.0 & $\mathbf{9 . 1}$ & 5 \\
\hline
\end{tabular}

Although the vector-loop morphologies were quite different from those observed during uniform propagation (compare loops in fig. 3 with loop in fig. 2), the basic properties of $\mathbf{E}$ were preserved, that is, the peak field $\hat{\mathbf{E}}$ represents a precise measure for activation time and direction of propagation.

\section{Acknowledgements}

This work was supported in part by grants from the Austrian Science Research Fund (E. Schrödinger Fellowship J-2132-INF), and by the Plan Nacional de Investigación Científica, Desarrollo e Innovación Tecnológica del Ministerio de Ciencia y Tecnología of Spain (TIC 2001-2686).

\section{References}

[1] Plank G, Hofer E. Model study of vector-loop morphology during electrical mapping of microscopic conduction in cardiac tissue. Ann Biomed Eng 2000;28:1244-1252.

[2] Kadish AH, Spear JF, Levine JH, Hanich R F amd Prood C, Moore EN. Vector mapping of myocardial activation. Circulation 1986;74:603-615.

[3] Witkowski FX, Kavanagh KM, Penkoske PA, Plonsey R. In vivo estimation of cardiac transmembrane current. Circ Res 1993;72:424-439.

[4] Nygren A, Fiset C, Firek L, Clark JW, Lindblad DS, Clark RB, Giles WR. Mathematical model of an adult human atrial cell: the role of $\mathrm{k}+$ currents in repolarization. Circ Res 1998; 82:63-81.

[5] Spach MS, Kootsey JM. Relating the sodium current and conductance to the shape of transmembrane and extracellular potentials by simulation: effects of propagation boundaries. IEEE Trans Biomed Eng 1985;32:743-755.

[6] Bayly PV, KenKnight BH, Rogers JM, Hillsley RE, Ideker RE, Smith WM. Estimation of conduction velocity vector fields from epicardial mapping data. IEEE Trans Biomed Eng 1998;45:563-571.

Address for correspondence:

Gernot Plank

Medizinische Physik und Biophysik / Universität Graz

Harrachgasse 21/ A-8010 Graz / Austria

tel./fax: ++43-316-380-4154/9660

gernot.plank@uni-graz.at 